\title{
ADVERSE REPRODUCTIVE HEALTH EFFECTS OF EXPOSURE TO CHLORINATION DISINFECTION BY PRODUCTS
}

\section{M.J. NIEUWENHUIJSEN*}

Received: $10 / 01 / 05$

Accepted: 30/03/05

\author{
Department of Epidemiology and Public Health \\ Division of Primary Care and Population Health Sciences \\ Faculty of Medicine, Imperial College London \\ St Mary's Campus, Norfolk Place, London, W2 1 PG, U.K.
}

\begin{abstract}
Chlorination disinfection by-products (DBPs) are formed when water is chlorinated and the organic matter in the water reacts with chlorine to form these by-products. There have been concerns about the potential health effects of these by-products, including cancer and reproductive effects. Here we have reviewed the literature on reproductive health effects. Epidemiological studies on neural tube defects, urinary tract defects and small for gestation age/intra growth retardation have shown the most consistent statistically significant associations with an index of DBPs, but generally the risk estimates are small. The interpretation of the studies is not straight forward because they may not be directly comparable because of differences in DBP mixtures, exposure categories and actually uptake of DBPs due to differences in e.g. ingestion rates, showering, bathing, and swimming. Only few specific DBPs have been studied and THMs have often been used as a marker for other DBPs, since they are often routinely available. Sample sizes, and therefore power, have at times been low, particularly when the population was split into exposure categories. Although most studies considered some confounders, (residual) confounding by other water contaminants or other factors related to water intake, cannot always be excluded. Case ascertainment, for outcomes such as spontaneous abortion and certain congenital anomalies is far from straight forward, and for the latter at times anomalies are lumped together with different aetiology, which may be inappropriate. Furthermore, as with many reproductive epidemiological, if the putative agent affects both early pregnancy loss and later birth outcomes such as congenital anomalies, interpretation of later birth outcomes may be more difficult.
\end{abstract}

KEYWORDS: Chlorination by products, trihalomethane, reproductive health effects, cancer

\section{INTRODUCTION}

Chlorination disinfection by-products (DBPs) are formed when water is chlorinated and the organic matter in the water reacts with chlorine to form these by-products. The formation and occurrence depends on many factors, including the chlorine dose, type of treatment, $\mathrm{pH}$, temperature, residence time, bromine levels (Nieuwenhuijsen et al., 2000a, IPCS 2000). Up to 500 different by-products have been identified (Richardson, 1998). Different mixtures of by-product may exist in different locations depending on the various factors mentioned above, making it more difficult to assess any health effects of DBPs, particularly in epidemiological studies.

The health effects of DBPs in drinking water have been a concern since DBPs were first reported in the seventies (Rook, 1974). Early studies focused on cancer outcomes, while 
the more recent studies have focused on reproductive outcomes (IPCS 2000). According to the recent review by IPCS (2000): "more studies have considered bladder cancer than any other cancer. The authors of the most recently reported results for bladder cancer risks caution against a simple interpretation of the observed associations. The epidemiological evidence for an increased relative risk for bladder cancer is not consistent - different risks are reported for smokers and non-smokers, for men and women, and for low and high water consumption. Risk may differ among various geographic areas because the DBP mix may be different or because other water contaminants are also present. More comprehensive water quality data must be collected or simulated to improve exposure assessments for epidemiological studies." A recent pooled analysis by Villanueva et al (2004), that provided quantitative information, confirmed this. For men there was an exposure response related relationship between DBP intake and bladder cancer, but there was no relationship in women (Table 1).

Table 1. Pooled analysis of bladder cancer and THM*

\begin{tabular}{|lll|}
\hline THM & & \\
\hline Exposure & Male ORs & Females ORs \\
\hline Level & & \\
\hline & & \\
\hline $0-15 \mathrm{mg}$ & 1.00 & 1.00 \\
\hline$>15-50 \mathrm{mg}$ & 1.22 & 0.92 \\
\hline$>50-400 \mathrm{mg}$ & 1.28 & 0.94 \\
\hline$>400-1000 \mathrm{mg}$ & 1.31 & 1.02 \\
\hline$>1000 \mathrm{mg}$ & 1.50 & 0.92 \\
\hline * After Villanueva et al. 2004 & \\
\hline
\end{tabular}

Reproductive health outcomes should be easier to study from an exposure point of view, because of the shorter relevant exposure period. Amongst others, birth weight, prematurity, spontaneous abortion, congenital anomalies and still birth have been the focus of these studies. Various thorough reviews have been conducted and concluded that there are still many problems to overcome and that the results are inconsistent and inconclusive (Reif et al., 1996; Nieuwenhuijsen et al., 2000b; Gevecker Graves et al., 2001; Bove et al., 2002; IPCS 2000). Table 2 provides an overview of the main reproductive epidemiological studies, their study design, sample size, exposure indices, confounders and main positive outcomes.

A number of studies found statistically significant positive associations between THMs and neural tube defects, one of the most studied group of congenital anomalies (Bove et al., 1995; Klotz and Pyrch, 1999; Dodds et al., 2001), while others did not (Magnus et al., 1999; Kallen et al., 2000; Hwang et al., 2002; Dodds et al., 1999; Shaw et al., 2003). Klotz and Pyrch (1999) found a statistically significant association between TTHM levels in the water and neural tube defects, but not with haloacetonitriles and haloacetates. Also, the effects were most pronounced in offspring from women that did not take supplementary vitamins, but these findings were not confirmed by the Shaw et al. (2003) study. Inclusion of information on ingestion, showering, bathing and swimming made little difference to the risk estimates.

Hwang et al. (2002) and Cedergren et al. (2002) found significant associations between chlorinated water and levels of TTHM above $10 \mathrm{mg} \mathrm{I}^{-1}$ respectively and respiratory defects, but other studies did not find such an association (Magnus et al., 1999; Kallen et al., 2000; Bove et al., 1995; Dodds et al., 1999; Dodds et al., 2001; Shaw et al., 2003). Studies on chlorinated water and respiratory effects have been rare, but two studies found a significant positive association (Aschengrau et al., 1993 and Hwang et al., 2002). 
Table 2. Summary of epidemiological studies on chlorinated disinfection by-products and adverse reproductive outcomes.

\begin{tabular}{|c|c|c|c|c|c|}
\hline $\begin{array}{l}\text { Author } \\
\text { (year) }\end{array}$ & $\begin{array}{l}\text { Study details } \\
\text { (location, time, } \\
\text { sample size) }\end{array}$ & Cases & Exposure assessment & $\begin{array}{l}\text { Other risk } \\
\text { factors included }\end{array}$ & $\begin{array}{l}\text { Main positive findings } \\
\text { OR }(95 \% \mathrm{Cl})\end{array}$ \\
\hline $\begin{array}{l}\text { Aschengrau } \\
\text { et al., } 1989\end{array}$ & $\begin{array}{l}\text { Massachusetts., } \\
\text { US. } \\
\text { Sample } \\
\text { population } \\
1677\end{array}$ & $\begin{array}{l}286 \text { spontaneous } \\
\text { abortion }\end{array}$ & $\begin{array}{l}\text { Surface vs ground water } \\
\text { Chlorination vs } \\
\text { chloraminated water }\end{array}$ & $\begin{array}{l}\text { Smoking habits } \\
\text { Contraceptive use } \\
\text { Medical and } \\
\text { obstetrical history } \\
\text { Metals }\end{array}$ & $\begin{array}{l}\text { Surface vs Ground water } \\
2.2(1.3-3.6)\end{array}$ \\
\hline $\begin{array}{l}\text { Kramer et al. } \\
\text { [48]. (1992) }\end{array}$ & $\begin{array}{l}\text { lowa, US. } \\
151 \text { towns with a } \\
\text { single water } \\
\text { source. } \\
\text { 1989-90 } \\
\text { Sample } \\
\text { population: } 4028\end{array}$ & $\begin{array}{l}588 \text { (total) } \\
159 \text { low birth weight } \\
342 \text { pre-term delivery } \\
187 \text { Intrauterine } \\
\text { growth } \\
\text { retardation/small-for- } \\
\text { gestational-age }\end{array}$ & $\begin{array}{l}\text { Based on maternal } \\
\text { residential address and one } \\
\text { municipal water survey to } \\
\text { estimate individual THM } \\
\text { levels } \\
\text { ( } 2 \text { or } 3 \text { exposure categories). }\end{array}$ & $\begin{array}{l}\text { Maternal age, } \\
\text { Parity, } \\
\text { Marital status, } \\
\text { Education, } \\
\text { Smoking, } \\
\text { Prenatal care. }\end{array}$ & $\begin{array}{l}\text { No vs Medium }\left(1-9 \mu \mathrm{g}^{-1}\right) \text { vs } \\
\text { High }\left(=>10 \mu \mathrm{I}^{-1}\right) \text { : } \\
\text { Chloroform } \\
\text { Low birth weight: } \\
1 \text { vs } 1.1(0.7-1.6) \text { vs } 1.3(0.8-2.2) \\
\text { Intrauterine growth retardation } \\
1 \text { vs } 1.3(0.9-1.8) \text { vs } 1.8(1.1-2.9) \\
\text { Dichlorobromomethane } \\
\text { Intrauterine growth retardation } \\
1 \text { vs } 1.2(0.8-1.7) \text { vs } 1.7(0.9-2.9)\end{array}$ \\
\hline $\begin{array}{l}\text { Aschengrau } \\
\text { et al. [45] } \\
(1993)\end{array}$ & $\begin{array}{l}\text { Massachusetts., } \\
\text { US. } \\
2 \text { hospitals. } \\
1977-80 \\
\text { Sample } \\
\text { population: } 2348\end{array}$ & $\begin{array}{l}1171 \text { (total) } \\
1039 \text { Major congenital } \\
\text { malformations } \\
\text { Urinary tract defects } \\
\text { Respiratory tract } \\
\text { defects } \\
77 \text { Stillbirths } \\
55 \text { Neonatal deaths }\end{array}$ & $\begin{array}{l}\text { Based on maternal } \\
\text { residential address to } \\
\text { ascertain type of water } \\
\text { supply, chlorination vs. } \\
\text { chloramination, and } \\
\text { ground/mixed water vs } \\
\text { surface water. }\end{array}$ & $\begin{array}{l}\text { Maternal age, } \\
\text { Pregnancy } \\
\text { history, } \\
\text { Alcohol, } \\
\text { Ethnicity, } \\
\text { Hospital payment, } \\
\text { Other water } \\
\text { contaminants. }\end{array}$ & 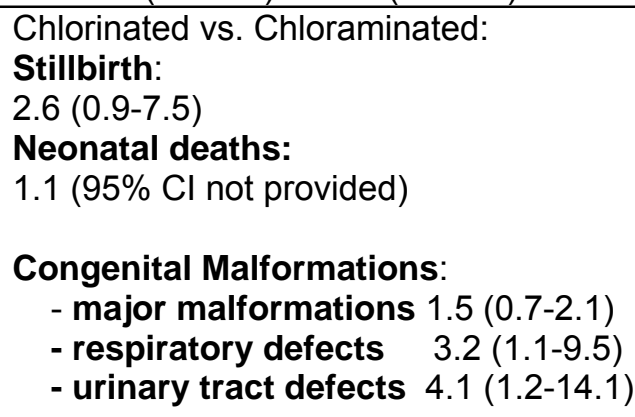 \\
\hline $\begin{array}{l}\text { Bove et al. } \\
\text { [49] (1995) }\end{array}$ & $\begin{array}{l}\text { New Jersey, US. } \\
75 \text { towns with a } \\
\text { public water } \\
\text { supply. } \\
1985-88 \\
\text { Sample } \\
\text { population: } 81602\end{array}$ & $\begin{array}{l}29268 \text { (total) } \\
\text { Live births: } \\
1853 \text { Low birth weight } \\
905 \text { Very low birth } \\
\text { weight } \\
4082 \text { Small-for- }\end{array}$ & $\begin{array}{l}\text { Based on maternal } \\
\text { residential address and } \\
\text { municipal water surveys to } \\
\text { estimate monthly TTHM } \\
\text { levels ( } 5 \text { or } 6 \text { exposure } \\
\text { categories) }\end{array}$ & $\begin{array}{l}\text { Maternal age } \\
\text { Ethnicity } \\
\text { Gender baby } \\
\text { Primipara } \\
\text { Prenatal care, } \\
\text { Education, } \\
\text { Previous still or }\end{array}$ & $\begin{array}{l}\text { TTHM levels }>100 \mu \mathrm{gl}^{-1} \mathrm{vs}<=20 \mu \mathrm{g} \mathrm{I}^{-1}: \\
\text { Low birth weight: } \\
1.4(50 \% \mathrm{Cl} 1.2-1.7) \\
\text { Intrauterine growth retardation/ } \\
\text { Small-for-gestational-age: } \\
1.5(90 \% \mathrm{Cl} 1.2-1.9)\end{array}$ \\
\hline
\end{tabular}


Table 2. (continued)

\begin{tabular}{|c|c|c|c|c|c|}
\hline $\begin{array}{l}\text { Author } \\
\text { (year) }\end{array}$ & $\begin{array}{l}\text { Study details } \\
\text { (location, time, } \\
\text { sample size) }\end{array}$ & Cases & Exposure assessment & $\begin{array}{l}\text { Other risk } \\
\text { factors included }\end{array}$ & $\begin{array}{l}\text { Main positive findings } \\
\text { OR }(95 \% \mathrm{CI})\end{array}$ \\
\hline & & $\begin{array}{l}\text { gestational-age } \\
7167 \text { Pre-term } \\
594 \text { Foetal deaths } \\
\text { All births: Defects: } \\
669 \text { Surveillance } \\
118 \text { Central nervous } \\
\text { system defects } \\
83 \text { Oral cleft } \\
56 \text { Neural Tube } \\
108 \text { Major cardiac }\end{array}$ & & $\begin{array}{l}\text { miscarriage } \\
\text { Other } \\
\text { contaminants }\end{array}$ & $\begin{array}{l}\text { TTHM levels >80 } \mu \mathrm{gl}^{-1} \mathrm{vs}<=20 \mu \mathrm{gl}^{-1}: \\
\text { Surveillance Register defects } \\
1.6(90 \% \mathrm{Cl} 1.2-2.0) \\
\text { CNS system defects: } \\
2.6(90 \% \mathrm{Cl} 1.5-4.3) \\
\text { Neural tube defects: } \\
3.0(90 \% \mathrm{Cl} 1.3-6.6) \\
\text { Major cardiac defects: } \\
1.8(90 \% \mathrm{Cl} 1.0-3.3) \\
\text { TTHM levels >100 } \mu \mathrm{g} \mathrm{I}^{-1} \text { vs }<=20 \mu \mathrm{g} \mathrm{I}^{-1} \text { : } \\
\text { Oral cleft defects: } \\
\text { 3.2 (90\%Cl 1.2-7.3) }\end{array}$ \\
\hline $\begin{array}{l}\text { Savitz et al. } \\
\text { [52] (1995) }\end{array}$ & $\begin{array}{l}\text { Carolina, US. } \\
6 \text { hospitals. } \\
1988-91 \\
\text { Sample } \\
\text { population: } 1003\end{array}$ & $\begin{array}{l}548 \text { (Total) } \\
126 \text { Spontaneous } \\
\text { Abortion } \\
244 \text { Pre-term } \\
178 \text { Low birth weight }\end{array}$ & $\begin{array}{l}\text { Based on maternal } \\
\text { residential address and } \\
\text { quarterly municipal water } \\
\text { surveys to estimate average } \\
\text { TTHM levels. } \\
\text { Analysis of: } \\
\text { a) surface vs ground water } \\
\text { source, } \\
\text { b) TTHM levels, } \\
\text { (3 exposure categories) } \\
\text { c) Consumption during } \\
\text { pregnancy } \\
\text { d) water source } x \text { amount } \\
\text { e) TTHM dose (level x } \\
\text { amount) }\end{array}$ & $\begin{array}{l}\text { Maternal age } \\
\text { Ethnicity } \\
\text { Hospital } \\
\text { Education } \\
\text { Marital status } \\
\text { Poverty level } \\
\text { Smoking } \\
\text { Alcohol } \\
\text { consumption. } \\
\text { Employment } \\
\text { Nausea }\end{array}$ & $\begin{array}{l}\text { 40.8-59.9 vs. } 81.1-168.8 \mu \mathrm{g} \mathrm{I}^{-1} \text { TTHM: } \\
\text { Spontaneous Abortion: } \\
1.2(0.6-2.4) \\
40.8-63.3 \text { vs. } 82.8-168.8 \mu \mathrm{g} \mathrm{I}^{-1} \text { TTHM: } \\
\text { Low birth weight: } \\
1.3(0.8-2.1) \\
\text { Per } 50 \mu \mathrm{g} \mathrm{I}^{-1} \text { TTHM increment change: } \\
\text { Spontaneous Abortion: } \\
1.7(1.1-2.7)\end{array}$ \\
\hline $\begin{array}{l}\text { Kanitz et al. } \\
\text { [46] (1996) }\end{array}$ & $\begin{array}{l}\text { Liguria, Italy. } \\
2 \text { hospitals. } \\
1988-1989 \\
\text { Sample } \\
\text { population: } 676\end{array}$ & $\begin{array}{l}548 \text { live births in } \\
\text { 'exposed' area. } \\
50 \text { Pre-term } \\
141 \text { Caesarean } \\
\quad \text { section } \\
133 \text { Neonatal jaundice }\end{array}$ & $\begin{array}{l}\text { Based on maternal } \\
\text { residential address to } \\
\text { ascertain type of water } \\
\text { source (chlorine dioxide +/or } \\
\text { hypochlorite vs. not treated). }\end{array}$ & $\begin{array}{l}\text { Maternal age } \\
\text { Education } \\
\text { Smoking } \\
\text { Alcohol } \\
\text { Gender of child }\end{array}$ & $\begin{array}{l}\text { Sodium hypochlorite treated }\left(8-16 \mu \mathrm{I}^{-1}\right. \\
\text { TTHMs) vs non treated water: } \\
\text { Neonatal jaundice } \\
1.1(0.7-2.8) \\
\text { Low birth weight } \\
6.0(0.6-12.6)\end{array}$ \\
\hline
\end{tabular}


Table 2. (continued)

\begin{tabular}{|c|c|c|c|c|c|}
\hline $\begin{array}{l}\text { Author } \\
\text { (year) }\end{array}$ & $\begin{array}{l}\text { Study details } \\
\text { (location, time, } \\
\text { sample size) }\end{array}$ & Cases & Exposure assessment & $\begin{array}{l}\text { Other risk } \\
\text { factors included }\end{array}$ & $\begin{array}{l}\text { Main positive findings } \\
\text { OR }(95 \% \mathrm{Cl})\end{array}$ \\
\hline & & $\begin{array}{l}20 \text { Low birth weight } \\
288 \text { Small body length } \\
370 \text { Small cranial } \\
\text { circumference }\end{array}$ & & & $\begin{array}{l}\text { Small body length } \\
2.3(1.3-4.2) \\
\text { Small cranial circumference } \\
3.5(2.1-8.5)\end{array}$ \\
\hline $\begin{array}{l}\text { Waller et al. } \\
\text { [53] } \\
(1998)\end{array}$ & $\begin{array}{l}\text { California, US. } \\
3 \text { regions of } \\
\text { surface, ground } \\
\text { and mixed } \\
\text { drinking water } \\
\text { 1989-1991 } \\
\text { Sample } \\
\text { population: } 5144 \\
\text { pregnancies }\end{array}$ & $\begin{array}{l}499 \text { Spontaneous } \\
\text { Abortions }\end{array}$ & $\begin{array}{l}\text { Based on maternal } \\
\text { residential address and } \\
\text { quarterly municipal water } \\
\text { surveys to estimate average } \\
\text { TTHM and individual THM } \\
\text { levels. Analysis based on: } \\
\text { a) THM levels } \\
\text { (3 or } 10 \text { exposure categories) } \\
\text { b) consumption during first } \\
\quad \text { trimester from interview } \\
\text { ( } 2 \text { exposure categories) }\end{array}$ & $\begin{array}{l}\text { Maternal age, } \\
\text { Gestational age, } \\
\text { Smoking, } \\
\text { History of } \\
\text { pregnancy loss, } \\
\text { Ethnicity, } \\
\text { Employment }\end{array}$ & $\begin{array}{l}\text { high TTHM dose }\left(\geq 5 \text { glasses/day }+\geq 75 \mu \mathrm{g} \mathrm{I}^{-1}\right) \\
\text { vs. Iow dose }\left(<5 \text { glasses/day }+<75 \mu \mathrm{g} \mathrm{I}^{-1}\right): \\
\text { Spontaneous Abortion } \\
1.8(1.1-3.0) \text {. } \\
\text { high BDCM dose }\left(\geq 5 \text { glasses/day }+\geq 18 \mu \mathrm{g} \mathrm{I}^{-1}\right) \\
\text { vs. low dose }\left(<5 \text { glasses/day }+<18 \mu \mathrm{g} \mathrm{I}^{-1}\right): \\
\text { Spontaneous Abortion } \\
3.0(1.4-6.6) \text {. }\end{array}$ \\
\hline $\begin{array}{l}\text { Waller et al. } \\
(2001)\end{array}$ & $\begin{array}{l}\text { See Waller et al. } \\
{[53]} \\
(1998)\end{array}$ & $\begin{array}{l}\text { See Waller et al. [53] } \\
(1998)\end{array}$ & $\begin{array}{l}\text { See Waller et al. [53] } \\
\text { (1998) }\end{array}$ & $\begin{array}{l}\text { See Waller et al. } \\
{[53]} \\
(1998)\end{array}$ & $\begin{array}{l}\text { Re-analysis Waller et al. (1998) } \\
\text { Utility wide subset sample highest AOR } \\
\text { high TTHM dose }\left(\geq 5 \text { glasses/day }+\geq 75 \mu \mathrm{g} \mathrm{I}^{-1}\right) \\
\text { vs. low dose }\left(\geq 5 \text { glasses/day }+<75 \mu \mathrm{g} \mathrm{I}^{-1}\right) \text { : } \\
\text { Spontaneous Abortion } \\
5.1 \text { (1.8-14.7) } \\
\text { Little relationship with showering }\end{array}$ \\
\hline $\begin{array}{l}\text { Gallagher et } \\
\text { al. [50] (1998) }\end{array}$ & $\begin{array}{l}\text { Colorado, US. } \\
28 \text { census blocks } \\
\text { in } 2 \text { water } \\
\text { districts. } \\
\text { 1990-1993. } \\
\text { Sample } \\
\text { population: } \\
1244 \text { live births }\end{array}$ & $\begin{array}{l}72 \text { low birth weight } \\
29 \text { term-low birth } \\
\text { weight } \\
68 \text { pre-term delivery }\end{array}$ & $\begin{array}{l}\text { Based on maternal } \\
\text { residential address and } \\
\text { municipal water surveys. } \\
\text { Estimate of household TTHM } \\
\text { level during last trimester } \\
\text { based on hydraulic modelling } \\
\text { (4 exposure categories) }\end{array}$ & $\begin{array}{l}\text { Maternal age, } \\
\text { Smoking, } \\
\text { Marital status, } \\
\text { Parity, } \\
\text { Education, } \\
\text { Employment, } \\
\text { Pre-natal care }\end{array}$ & $\begin{array}{l}\text { high TTHM level }\left(=>61 \mu \mathrm{g} \mathrm{l}^{-1}\right) \mathrm{vs} \text {. lowest } \\
\left(<=20 \mu \mathrm{I}^{-1}\right): \\
\text { Low birth weight: } \\
2.1(1.0-4.8) \\
\text { Term low birth weight: } \\
5.9(2.0-17.0)\end{array}$ \\
\hline $\begin{array}{l}\text { Dodds et al. } \\
\text { [51] (1999) }\end{array}$ & $\begin{array}{l}\text { Nova Scotia, } \\
\text { Canada } \\
\text { 1988-1995 } \\
\text { Sample }\end{array}$ & $\begin{array}{l}4673 \text { Small for } \\
\text { gestational age } \\
2393 \text { Low birth weight } \\
342 \text { Very low birth }\end{array}$ & $\begin{array}{l}\text { Based on maternal } \\
\text { residential address and } \\
\text { TTHM levels for public water } \\
\text { facilities ( } 3 \text { sampling }\end{array}$ & $\begin{array}{l}\text { Maternal age } \\
\text { Parity } \\
\text { Maternal smoking } \\
\text { Attendance }\end{array}$ & $\begin{array}{l}0-49 \mu \mathrm{g} \mathrm{I}^{-1} \mathrm{vs}>100 \mu \mathrm{g} \mathrm{I}^{-1} \mathrm{TTHMs} \\
\text { Still birth } \\
1.66(1.09-2.52) \\
\text { Chromosomal abnormalities }\end{array}$ \\
\hline
\end{tabular}


Table 2. (continued)

\begin{tabular}{|c|c|c|c|c|c|}
\hline $\begin{array}{l}\text { Author } \\
\text { (year) }\end{array}$ & $\begin{array}{l}\text { Study details } \\
\text { (location, time, } \\
\text { sample size) }\end{array}$ & Cases & Exposure assessment & $\begin{array}{l}\text { Other risk } \\
\text { factors included }\end{array}$ & $\begin{array}{l}\text { Main positive findings } \\
\text { OR }(95 \% \mathrm{Cl})\end{array}$ \\
\hline & $\begin{array}{l}\text { population: } \\
49,842 \text { births }\end{array}$ & $\begin{array}{l}\text { weight } \\
2689 \text { pre-term delivery } \\
77 \text { Neural tube } \\
82 \text { Cleft defect } \\
430 \text { Major cardiac } \\
\text { defects } \\
197 \text { Still birth } \\
96 \text { chromosomal } \\
\text { abnormalities }\end{array}$ & $\begin{array}{l}\text { locations) modelled using } \\
\text { linear regression on the basis } \\
\text { of observations by year, } \\
\text { month and facility (4 } \\
\text { exposure categories) }\end{array}$ & $\begin{array}{l}\text { prenatal classes } \\
\text { Neighbourhood } \\
\text { family income } \\
\text { gender } \\
\text { Pregnancy and } \\
\text { pre-delivery } \\
\text { weight }\end{array}$ & $\begin{array}{l}1.38(0.73-2.59) \\
\text { Small for gestation age } \\
1.08(0.99-1.18) \\
\text { NTDs } \\
1.18(0.67-2.10)\end{array}$ \\
\hline $\begin{array}{l}\text { King et al. } \\
(2000)\end{array}$ & $\begin{array}{l}\text { Nova Scotia, } \\
\text { Canada } \\
1988-1995 \\
\text { Sample } \\
\text { population: } \\
49,756\end{array}$ & $\begin{array}{l}214 \text { still births } \\
\text { (72 asphyxia related } \\
\text { still births) }\end{array}$ & $\begin{array}{l}\text { Based on maternal } \\
\text { residential address and } \\
\text { TTHM, chloroform, and } \\
\text { BDCM levels for public water } \\
\text { facilities ( } 3 \text { sampling } \\
\text { locations) modelled using } \\
\text { linear regression on the basis } \\
\text { of observations by year, } \\
\text { month and facility ( } 4 \\
\text { exposure categories) } \\
(r=0.44 \text { for TTHM and } \\
\text { BDCM) }\end{array}$ & \begin{tabular}{|l|} 
Maternal age \\
Parity \\
Maternal smoking \\
Attendance \\
prenatal classes \\
Neighbourhood \\
family income \\
gender \\
Pregnancy and \\
pre-delivery \\
weight
\end{tabular} & $\begin{array}{l}0-49 \mu \mathrm{g} \mathrm{I}^{-1} \mathrm{vs}>100 \mu \mathrm{g} \mathrm{I}^{-1} \text { chloroform } \\
\text { Still birth } \\
1.56(1.04-2.34) \\
\text { Asphyxia related stillbirth } \\
3.15(1.64-6.03) \\
\quad 5 \mu \mathrm{g} \mathrm{I}^{-1} \mathrm{vs}>20 \mu \mathrm{g}^{-1} \mathrm{BDCM} \\
\text { Still birth } \\
1.98(1.23-3.49) \\
\text { Asphyxia related stillbirth } \\
1.75(0.72-4.22)\end{array}$ \\
\hline $\begin{array}{l}\text { Dodds and } \\
\text { King (2001) }\end{array}$ & $\begin{array}{l}\text { Nova Scotia, } \\
\text { Canada } \\
\text { 1988-1995 } \\
\text { Sample } \\
\text { population: } \\
49,842 \text { births }\end{array}$ & $\begin{array}{l}77 \text { NTDs } \\
430 \text { Cardiovascular } \\
\text { anomalies } \\
82 \text { Cleft defects } \\
96 \text { Chromosomal } \\
\text { abnormalities }\end{array}$ & $\begin{array}{l}\text { Based on maternal } \\
\text { residential address and } \\
\text { TTHM, chloroform, and } \\
\text { BDCM levels for public water } \\
\text { facilities ( } 3 \text { sampling } \\
\text { locations) modelled using } \\
\text { linear regression on the basis } \\
\text { of observations by year, } \\
\text { month and facility ( } 4 \\
\text { exposure categories) } \\
(r=0.44 \text { for TTHM and } \\
\text { BDCM) }\end{array}$ & $\begin{array}{l}\text { Maternal age } \\
\text { Parity } \\
\text { Maternal smoking } \\
\text { Attendance } \\
\text { prenatal classes } \\
\text { Neighbourhood } \\
\text { family income } \\
\text { gender } \\
\text { Pregnancy and } \\
\text { pre-delivery } \\
\text { weight }\end{array}$ & $\begin{array}{l}\mathrm{BDCM} \geq 20 \mu \mathrm{g} \mathrm{I}^{-1} \mathrm{vs}<5 \mu \mathrm{gl}^{-1} \\
\text { NTDs } \\
2.5(1.2-5.1)\end{array}$ \\
\hline $\begin{array}{l}\text { Klotz and } \\
\text { Pyrch [56] }\end{array}$ & $\begin{array}{l}\text { New Jersey, US } \\
\text { 1993-1994 }\end{array}$ & $\begin{array}{l}112 \text { Neural tube } \\
\text { defects }\end{array}$ & $\begin{array}{l}\text { Based on residential address } \\
\text { and public water facility }\end{array}$ & $\begin{array}{l}\text { Sociodemographi } \\
\text { cs }\end{array}$ & $\begin{array}{l}\text { TTHMs public monitoring data, } \\
\text { Known residence\&isolated cases }\end{array}$ \\
\hline
\end{tabular}


Table 2. (continued)

\begin{tabular}{|c|c|c|c|c|c|}
\hline $\begin{array}{l}\text { Author } \\
\text { (year) }\end{array}$ & $\begin{array}{l}\text { Study details } \\
\text { (location, time, } \\
\text { sample size) }\end{array}$ & Cases & Exposure assessment & $\begin{array}{l}\text { Other risk } \\
\text { factors included }\end{array}$ & $\begin{array}{l}\text { Main positive findings } \\
\text { OR }(95 \% \mathrm{Cl})\end{array}$ \\
\hline (1999) & $\begin{array}{l}\text { Sample } \\
\text { population: } \\
\text { All births, of which } \\
112 \text { cases- } 248 \\
\text { controls selected } \\
\end{array}$ & & $\begin{array}{l}\text { TTHM data, and tap water } \\
\text { sampling for TTHMs, HANs } \\
\text { and HAAs } \\
\text { (3-5 exposure categories) }\end{array}$ & $\begin{array}{l}\text { Pregnancy and } \\
\text { medical history } \\
\text { Parental } \\
\text { occupational } \\
\text { Use of vitamins } \\
\end{array}$ & $\begin{array}{l}<5 \mu \mathrm{gl}^{-1} \text { vs } 40+\mu \mathrm{g} \mathrm{I}^{-1} \\
\text { NTDs } \\
2.1(1.1-4.0)\end{array}$ \\
\hline $\begin{array}{l}\text { Magnus et al. } \\
{[47]} \\
(1999)\end{array}$ & $\begin{array}{l}\text { Norway } \\
\text { Sample } \\
\text { population: } \\
141,077\end{array}$ & $\begin{array}{l}\text { 2,608 all birth defects } \\
62 \text { Neural tube defects } \\
250 \text { Major cardiac } \\
\text { defects } \\
91 \text { Respiratory defects } \\
122 \text { Urinary defects } \\
143 \text { Oral cleft }\end{array}$ & $\begin{array}{l}\text { Chlorination yes vs no } \\
\text { Colour high vs low } \\
\text { (in chlorinated water average } \\
\text { TTHMs = 9.4 } \mu \mathrm{g} \mathrm{I}^{-1} \text {, average } \\
\text { HAAs }=14.6 \mu \mathrm{gl}^{-1} \text { ) }\end{array}$ & $\begin{array}{l}\text { Maternal age } \\
\text { Parity } \\
\text { Geographical } \\
\text { placement } \\
\text { Population } \\
\text { density } \\
\text { Industry profile }\end{array}$ & $\begin{array}{l}\text { No chlorination low colour vs } \\
\text { chlorination high colour } \\
\text { All birth defects } \\
1.14(0.99-1.31) \\
\text { Urinary tract defects } \\
1.99(1.10-3.57) \\
\text { NTDs } \\
1.26(0.61-2.62) \\
\text { Major cardiac defects } \\
1.05(0.76-1.46) \\
\text { Respiratory tract defects } \\
1.07(0.52-2.19) \\
\end{array}$ \\
\hline $\begin{array}{l}\text { Jaakkola et } \\
\text { al. }(2001)\end{array}$ & $\begin{array}{l}\text { Norway } \\
\text { Sample } \\
\text { population: } \\
137,145\end{array}$ & $\begin{array}{l}\text { 6249 Low birth weight } \\
\text { ? Small for gestational } \\
\text { age } \\
7886 \text { Pre-term delivery }\end{array}$ & $\begin{array}{l}\text { Chlorination yes vs no } \\
\text { Colour high vs low } \\
\text { (in chlorinated water average } \\
\text { TTHMs = 9.4 } \mu \mathrm{g} \mathrm{I}^{-1} \text {, average } \\
\text { HAAs }=14.6 \mu \mathrm{gl}^{-1} \text { ) }\end{array}$ & \begin{tabular}{|l|} 
Maternal age \\
Parity \\
Geographical \\
placement \\
Population \\
density \\
Industry profile \\
\end{tabular} & $\begin{array}{l}\text { No chlorination low colour vs } \\
\text { chlorination high colour } \\
\text { Pre-term delivery } \\
0.91(0.84-0.99)\end{array}$ \\
\hline $\begin{array}{l}\text { Kallen et al. } \\
(2000)\end{array}$ & $\begin{array}{l}\text { Sweden ('85-'94) } \\
\text { Sample } \\
\text { population: } \\
\text { No chlorination: } \\
74324 \text { singletons } \\
\text { Na-hypochlorite: } \\
\text { 24,731 singletons } \\
\text { Chlorine dioxide: } \\
\text { 15429 singletons }\end{array}$ & $\begin{array}{l}\text { Multiple births } \\
\text { Gestational duration } \\
\text { Birth weight } \\
\text { Intrauterine growth } \\
\text { Body length } \\
\text { Head circumference } \\
\text { Body mass index } \\
\text { Infant survival up to } 1 \\
\text { yr } \\
\text { Perinatal death } \\
\text { Apgar score }\end{array}$ & $\begin{array}{l}\text { No versus sodium } \\
\text { hypochlorite } \\
\text { (no versus chlorine dioxide) }\end{array}$ & $\begin{array}{l}\text { Year of birth } \\
\text { Maternal age } \\
\text { Parity } \\
\text { Maternal } \\
\text { education } \\
\text { Maternal smoking } \\
\text { Congenital } \\
\text { malformations } \\
\text { and childhood } \\
\text { cancer: }\end{array}$ & $\begin{array}{l}\text { No versus sodium hypochlorite } \\
\text { Low birth weight } \\
1.15(1.05-1.26) \\
<32 \text { weeks gestation } \\
1.22(1.00-1.48) \\
<37 \text { weeks gestation } \\
1.09(1.01-1.17) \\
<43 \mathrm{~cm} \text { length } \\
1.97(1.30-2.97) \\
<47 \mathrm{~cm} \text { length } \\
1.25(1.10-1.43)\end{array}$ \\
\hline
\end{tabular}


Table 2. (continued)

\begin{tabular}{|c|c|c|c|c|c|}
\hline $\begin{array}{l}\text { Author } \\
\text { (year) }\end{array}$ & $\begin{array}{l}\text { Study details } \\
\text { (location, time, } \\
\text { sample size) }\end{array}$ & Cases & Exposure assessment & $\begin{array}{l}\text { Other risk } \\
\text { factors included }\end{array}$ & $\begin{array}{l}\text { Main positive findings } \\
\text { OR }(95 \% \mathrm{Cl})\end{array}$ \\
\hline & & $\begin{array}{l}\text { Neonatal jaundice } \\
\text { Congenital } \\
\text { malformations, incl } \\
\text { NTD } \\
\text { Childhood cancer } \\
\text { Hypothyroidism }\end{array}$ & & \begin{tabular}{|l|} 
Maternal age \\
Year of birth
\end{tabular} & $\begin{array}{l}\text { BMI>16 } \mathbf{~ k ~ ~}^{-2} \\
1.27(1.19-1.37) \\
<31 \mathbf{c m} \text { head circumference } \\
1.46(1.07-1.98) \\
\text { Spine malformation } \\
3.2(1.0-10.0)\end{array}$ \\
\hline $\begin{array}{l}\text { Yang et al. } \\
(2000 a)\end{array}$ & $\begin{array}{l}\text { Taiwan } \\
\text { Sample } \\
\text { population } \\
18,025 \text { first parity } \\
\text { births: } \\
\text { Chlorinated: } \\
10,007 \\
\text { Non-chlorinated: } \\
8,018 \\
\end{array}$ & $\begin{array}{l}\text { Low birth weight } \\
\text { Preterm delivery }(<37 \\
\text { weeks) }\end{array}$ & $\begin{array}{l}\text { Chlorinated }(>95 \% \text { pop } \\
\text { served chlorin. water) versus } \\
\text { non-chlorinated }(<5 \% \text { pop } \\
\text { served chlorin. water })\end{array}$ & $\begin{array}{l}\text { Maternal age } \\
\text { Marital status } \\
\text { Maternal } \\
\text { education } \\
\text { Gender }\end{array}$ & $\begin{array}{l}\text { Chlorinated versus non-chlorinated } \\
\text { Preterm delivery } \\
1.34(1.15-1.56)\end{array}$ \\
\hline $\begin{array}{l}\text { Yang et al. } \\
\text { (2000b) }\end{array}$ & $\begin{array}{l}\text { Taiwan } \\
\text { Sample } \\
\text { population } \\
\text { Chlorinated: } \\
24882 \\
\text { Non-chlorinated: } \\
20460\end{array}$ & Sex ratio & $\begin{array}{l}\text { Chlorinated }(>95 \% \text { pop } \\
\text { served chlorin. water) versus } \\
\text { non-chlorinated }(<5 \% \text { pop } \\
\text { served chlorin. water })\end{array}$ & & \\
\hline $\begin{array}{l}\text { Cedergren et } \\
\text { al. } \\
(2002)\end{array}$ & $\begin{array}{l}\text { Sweden } \\
\text { Sample } \\
\text { population } \\
58669\end{array}$ & Cardiac defects & $\begin{array}{l}>10 \mu \mathrm{gl}^{-1} \mathrm{vs} \leq 10 \mu \mathrm{gl}^{-1} \\
\text { TTHM in surface water } \\
\text { hypochloride and chlorine } \\
\text { dioxide vs hypochloride in } \\
\text { surface water } \\
\text { Ground water vs surface } \\
\text { water }\end{array}$ & \begin{tabular}{|l|} 
Maternal age \\
Parity \\
Smoking \\
Education
\end{tabular} & $\begin{array}{l}>10 \mu \mathrm{g} \mathrm{I}^{-1} \mathrm{vs} \leq 10 \mu \mathrm{g} \mathrm{I}^{-1} \mathrm{TTHM} \\
\text { Cardiac defects } \\
1.30(1.08-1.56) \\
\text { Ground water vs surface water } \\
\text { Cardiac defects } \\
1.32(1.10-1.58) \\
\\
\text { Hypochloride and chlorine dioxide vs } \\
\text { hypochloride } \\
\text { Cardiac defects } \\
1.85(1.42-2.39)\end{array}$ \\
\hline
\end{tabular}


Table 2. (continued)

\begin{tabular}{|c|c|c|c|c|c|}
\hline $\begin{array}{l}\text { Author } \\
\text { (year) }\end{array}$ & $\begin{array}{l}\text { Study details } \\
\text { (location, time, } \\
\text { sample size) }\end{array}$ & Cases & Exposure assessment & $\begin{array}{l}\text { Other risk } \\
\text { factors included }\end{array}$ & $\begin{array}{l}\text { Main positive findings } \\
\text { OR }(95 \% \mathrm{Cl})\end{array}$ \\
\hline $\begin{array}{l}\text { Hwang et al., } \\
\text { (2002) }\end{array}$ & $\begin{array}{l}\text { Norway } \\
\text { Sample } \\
\text { population } \\
285,631\end{array}$ & $\begin{array}{l}\text { Any birth defect } \\
\text { Neural tube defect } \\
\text {-Anencephalus } \\
\text {-Spina bifida } \\
\text {-hydrocephalus } \\
\text { Cardiac defects } \\
\text {-Ventricular septal } \\
\text { defects } \\
\text {-Atrial septal defects } \\
\text { Respiratory defects } \\
\text { Oral cleft defects } \\
\text { Cleft palate } \\
\text { Cleft lip } \\
\text { Urinary tract defect } \\
\text {-Obstructive urinary } \\
\text { tract defect }\end{array}$ & $\begin{array}{l}\text { Chlorination (yes/no) and } \\
\text { level of water colour }(\mathrm{mg} \mathrm{Pt} \\
\left.\mathrm{I}^{-1}:<10,10-19.9, \geq 20\right)\end{array}$ & $\begin{array}{l}\text { Maternal age } \\
\text { Parity } \\
\text { Socioeconomic } \\
\text { status: } \\
\text {-Centrality } \\
\text {-Population } \\
\text { density }\end{array}$ & $\begin{array}{l}\text { Chlorination (yes) and level of water colour: } \\
<10 \text { vs } \geq 20 \mathrm{mg} \mathrm{Pt} l^{-1} \\
\text { All birth defect } \\
1.18(1.02-1.36) \\
\text { Ventricular septal defect } \\
1.81 \text { (1.05-3.09) } \\
\text { Chlorination (yes) and level of water colour: } \\
<10 \text { vs } \geq\left. 10 \mathrm{mg} \mathrm{Pt}\right|^{-1} \\
\\
\text { All birth defects } \\
1.13(1.01-1.25) \\
\text { Cardiac defects } \\
1.37(1.00-1.89) \\
\text { Respiratory defects } \\
1.89(1.00-3.58) \\
\text { Urinary tract defects } \\
1.46(1.00-2.13)\end{array}$ \\
\hline $\begin{array}{l}\text { Nieuwenhuijs } \\
\text { en et al. } \\
\text { (2002) }\end{array}$ & $\begin{array}{l}\text { England } \\
11,462\end{array}$ & Birth weight & Amount of swimming (hrs) & $\begin{array}{l}\text { Maternal age } \\
\text { Maternal } \\
\text { education } \\
\text { Smoking } \\
\text { Alcohol use } \\
\text { Drugs use } \\
\text { Gestational age } \\
\text { Ethnicity } \\
\text { Infant gender }\end{array}$ & \\
\hline $\begin{array}{l}\text { Wright et al. } \\
(2003)\end{array}$ & $\begin{array}{l}\text { Massachusetts, } \\
\text { USA } \\
56,513\end{array}$ & $\begin{array}{l}\text { Birth weight } \\
\text { Low birth weight } \\
\text { Small for gestational } \\
\text { age } \\
\text { Gestational age } \\
\text { Preterm delivery }\end{array}$ & $\begin{array}{l}0-60,>60-80,>80 \mu \mathrm{g} \mathrm{I}^{-1} \\
\text { TTHM or per } 20 \mu \mathrm{g} \mathrm{I}^{-1} \mathrm{TTHM} \\
\text { increase }\end{array}$ & $\begin{array}{l}\text { Maternal age } \\
\text { Maternal } \\
\text { education } \\
\text { Ethnicity } \\
\text { Smoking } \\
\text { Parental care } \\
\text { Parity } \\
\text { Infant gender }\end{array}$ & $\begin{array}{l}0-60, \text { vs }>80 \mu \mathrm{gl}^{-1} \text { TTHM } \\
\text { Birth weight } \\
-32 \mathrm{~g}(-47 \text { to }-18) \\
\text { Small for gestational age } \\
1.14(1.02-1.26) \\
\text { Gestational age (wks) } \\
0.08(0.01-0.14)\end{array}$ \\
\hline
\end{tabular}


Table 2. (continued)

\begin{tabular}{|c|c|c|c|c|c|}
\hline $\begin{array}{l}\text { Author } \\
\text { (year) }\end{array}$ & $\begin{array}{l}\text { Study details } \\
\text { (location, time, } \\
\text { sample size) } \\
\end{array}$ & Cases & Exposure assessment & $\begin{array}{l}\text { Other risk } \\
\text { factors included }\end{array}$ & $\begin{array}{l}\text { Main positive findings } \\
\text { OR }(95 \% \mathrm{Cl})\end{array}$ \\
\hline & & & & & $\begin{array}{l}\text { per } 20 \mu \mathrm{g} \mathrm{I}^{-1} \text { TTHM increase } \\
\text { Birth weight } \\
-2.8 \mathrm{~g}(-5.5 \text { to }-0.2) \\
\text { Gestational age } \\
0.02(0.01-0.03)\end{array}$ \\
\hline $\begin{array}{l}\text { Shaw et al. } \\
(2003)\end{array}$ & $\begin{array}{l}\text { California USA } \\
\text { Study 1: } 538 \text { NTD } \\
\text { cases and } 539 \\
\text { controls } \\
\text { Study 2: } 265 \text { NTD } \\
\text { cases, } 207 \\
\text { conotruncal heart } \\
\text { defect cases and } \\
409 \text { orofacial cleft } \\
\text { cases and } 481 \\
\text { controls }\end{array}$ & $\begin{array}{l}\text { Study 1: NTDs } \\
\text { (anencephaly and } \\
\text { spina bifida) } \\
\text { Study 2: NTDs } \\
\text { (anencephaly and } \\
\text { spina bifida), } \\
\text { conotruncal heart } \\
\text { defects, orofacial clefts }\end{array}$ & 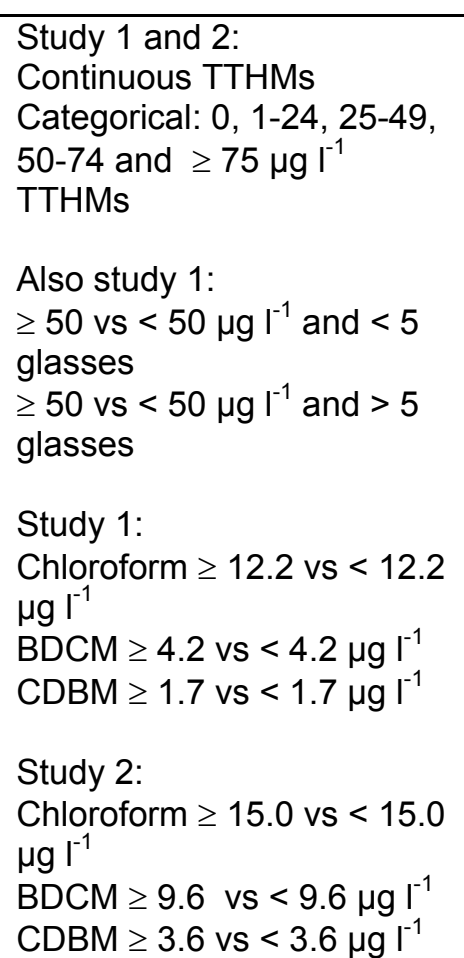 & $\begin{array}{l}\text { Ethnicity } \\
\text { Education } \\
\text { Body mass index } \\
\text { Use of vitamins } \\
\text { Methylenetetrahy } \\
\text { drofolate } \\
\text { reductase } \\
\text { (MTHFR) } \\
\text { genotype }\end{array}$ & $\begin{array}{l}\text { Study 1: NTDs } \\
\text { NTD risk inversely related to TTHM exposure } \\
\text { but only occasionally significant for one } \\
\text { category } \\
\text { Chloroform } \geq 12.2 \mathrm{vs}<12.2 \mu \mathrm{g} \mathrm{I}^{-1} \\
0.50(0.34-0.75) \\
\text { BDCM } \geq 4.2 \mathrm{vs}<4.2 \mu \mathrm{gl}^{-1} \\
0.66(0.45-0.97) \\
\text { CDBM } \geq 1.7 \mathrm{vs}<1.7 \mu \mathrm{g} \mathrm{I}^{-1} \\
0.69(0.47-1.0) \\
\text { Study } 2 \text { : Multiple cleft palate/lip } \\
\text { Chloroform } \geq 15.0 \mathrm{vs}<15.0 \mu \mathrm{g} \mathrm{I}^{-1} \\
0.21(0.05-0.90)\end{array}$ \\
\hline $\begin{array}{l}\text { Dodds et al. } \\
(2004)\end{array}$ & $\begin{array}{l}\text { Nova Scotia and } \\
\text { Eastern Ontario } \\
112 \text { stillbirth and } \\
398 \text { live birth } \\
\text { controls }\end{array}$ & Still birth & $\begin{array}{l}\text { Various indices: } \\
0,1-49,50-79 \text { and }>80 \mu \mathrm{g} \mathrm{I}^{-1} \\
\text { for total THMs and } \\
\text { chloroform and } \\
0,1-4,5-9 \text { and }>9 \text { for BDCM }\end{array}$ & $\begin{array}{l}\text { Age } \\
\text { Province } \\
\text { Household } \\
\text { income }\end{array}$ & $\begin{array}{l}\text { Still birth: } \\
\text { TTHM >80 vs } 0 \\
2.2(1.1-4.4) \\
\text { TTHM highest vs lowest quintile } \\
2.4(1.2-4.6)\end{array}$ \\
\hline
\end{tabular}


Table 2. (continued)

\begin{tabular}{|c|c|c|c|c|c|}
\hline $\begin{array}{l}\text { Author } \\
\text { (year) }\end{array}$ & $\begin{array}{l}\text { Study details } \\
\text { (location, time, } \\
\text { sample size) }\end{array}$ & Cases & Exposure assessment & $\begin{array}{l}\text { Other risk } \\
\text { factors included }\end{array}$ & $\begin{array}{l}\text { Main positive findings } \\
\text { OR }(95 \% \mathrm{Cl})\end{array}$ \\
\hline & & & $\begin{array}{l}\text { Quintiles for total exposure } \\
\text { (ingestion/showring/bathing) } \\
\text { for TTHM, chloroform and } \\
\text { BDCM } \\
\text { Concentration and duration }\end{array}$ & & $\begin{array}{l}\text { Drinking } 5+\text { drinks per day and THM } 50+\mu \mathrm{I} \mathrm{I}^{-1} \\
\text { vs }<1 \text { drink and THM }=0 \\
4.0(1.4-11) \\
\text { Chloroform and BDCM generally follow TTHM } \\
\text { trend }\end{array}$ \\
\hline Yang (2004) & $\begin{array}{l}\text { Taiwan } \\
182,796\end{array}$ & $\begin{array}{l}\text { Low birth weight } \\
\text { Pre-term delivery }\end{array}$ & $\begin{array}{l}15 \text { non-chlorinating } \\
\text { municipalities (NCM) and } \\
128 \text { chlorinating } \\
\text { municipalities (CM) }\end{array}$ & $\begin{array}{l}\text { Maternal age } \\
\text { Education } \\
\text { Gestational age } \\
\text { Birth weight } \\
\text { Gender }\end{array}$ & $\begin{array}{l}\text { Pre-term delivery } \\
\text { NCM vs CM } \\
1.37(1.20-1.56)\end{array}$ \\
\hline $\begin{array}{l}\text { Infante- } \\
\text { Rivard (2004) }\end{array}$ & $\begin{array}{l}\text { Montreal, } \\
\text { Quebec, Canada } \\
493 \text { cases } \\
472 \text { control }\end{array}$ & $\begin{array}{l}\text { Intrauterine growth } \\
\text { restriction (10th \%ile) }\end{array}$ & $\begin{array}{l}\text { Regulatory data on THMs, }> \\
90^{\text {th }} \% \text { tile vs } \leq 90^{\text {th }} \% \text { tile }\end{array}$ & $\begin{array}{l}\text { Gestational age } \\
\text { Sex } \\
\text { Race } \\
\text { Mother's weight } \\
\text { gain } \\
\text { BMI } \\
\text { Smoking } \\
\text { Primiparity } \\
\text { Pre-eclampsia } \\
\text { Previous IUGR }\end{array}$ & $\begin{array}{l}\text { IUGR } \\
\text { No association with THMs only, but with } \\
\text { CYP2E1*5 (G1259C) } 13.2(1.19-146.7) \text { in } \\
\text { newborns }\end{array}$ \\
\hline $\begin{array}{l}\text { Wright et al. } \\
(2004)\end{array}$ & $\begin{array}{l}\text { Massachusetts, } \\
\text { US } \\
196,000 \text { registry } \\
\text { based }\end{array}$ & $\begin{array}{l}\text { birth weight } \\
\text { gestational age } \\
\text { small for gestation age } \\
\text { pre-term delivery }\end{array}$ & $\begin{array}{l}\text { TTHM } \\
\text { Ind THMs } \\
\text { HAAs } \\
\text { MX } \\
\text { Mutagenicity }\end{array}$ & & $\begin{array}{l}\text { SGA } \\
>74 \text { vs } \leq 33 \mu \mathrm{g} \mathrm{I}^{-1} \mathrm{TTHM} 1.13(1.07-1.20) \\
>63 \mathrm{vs} \leq 26 \mu \mathrm{g} \mathrm{I}^{-1} \mathrm{CHCL} 31.11(1.04-1.17) \\
>13 \mathrm{vs} \leq 5 \mu \mathrm{g} \mathrm{I}^{-1} \mathrm{BDCM} 1.15(1.08-1.22) \\
>2,250 \mathrm{vs} \leq 1250 \mathrm{rev} / \mathrm{l} \text { mutagenicity } 1.25 \\
(1.04-1.51) \\
\text { Similar results for birth weight }\end{array}$ \\
\hline $\begin{array}{l}\text { King et al. } \\
(2005)\end{array}$ & $\begin{array}{l}\text { Nova Scotia and } \\
\text { Eastern Ontario, } \\
\text { Canada } \\
112 \text { cases } \\
398 \text { controls } \\
\end{array}$ & stillbirth & HAAs & $\begin{array}{l}\text { Maternal age } \\
\text { Province } \\
\text { Income } \\
\text { Occupation } \\
\text { Smoking } \\
\end{array}$ & $\begin{array}{l}\text { Stillbirth } \\
\text { No significant results after adjustments for } \\
\text { THMs }\end{array}$ \\
\hline $\begin{array}{l}\text { Toledano et } \\
\text { al. (2005) }\end{array}$ & $\begin{array}{l}3 \text { water regions in } \\
\text { UK }\end{array}$ & $\begin{array}{l}\text { Low birth weight } \\
\text { Very low birth weight } \\
\text { Still birth }\end{array}$ & THMs & $\begin{array}{l}\text { Maternal age } \\
\text { Deprivation }\end{array}$ & $\begin{array}{l}\text { Stillbirth } \\
\geq 60 \text { vs }<\left.30 \mu \mathrm{g} \mathrm{I}\right|^{-1} \text { TTHM } 1.11(1.00-1.23)\end{array}$ \\
\hline
\end{tabular}


Similarly, for urinary tract defects, although only three studies have been conducted they all reported statistically significant associations (Aschengrau et al., 1993; Magnus et al., 1999, Hwang et al 2002). Studies on oral cleft or cleft palate have largely been negative, except for the study by Bove et al. (1995). In a meta-analysis Hwang et al. (2003) reported evidence for an effect of exposure chlorination by-products on the risk of neural tube and urinary system defects, but results for respiratory system, major cardiac and oral cleft defects were heterogeneous and inconclusive .

Only a few studies have assessed the relationship between DBPs and spontaneous abortion. The California study has attracted the most attention since they found a statistically significant association between TTHM and BDCM and spontaneous abortion (Waller et al., 1998). The effects were even stronger after re-analysis (Waller et al., 2001)

A number of Canadian studies and one English found statistically positive associations between DBPs and stillbirth (Dodds et al., 1999, King et al., 2000; Dodds et al., 2004; Toledano et al., 2005). However the case control study by Dodds et al., 2004 did not show a monotonic relationship between THM levels and stillbirth, and they did not find an association between HAAs and still birth (King et al., 2005).

Studies on pre-term delivery have generally shown no association with DBPs, with the exception of the study by Yang et al. (2000). Study results on low birth weight have been more mixed, with some studies reporting statistically significant associations (Kallen et al., 2000; Bove et al., 1995; Gallagher et al., 1998) while others did not find any statistically significant associations (Kanitz et al., 1996; Jaakkola et al., 2001; Kramer et al., 1992; Savitz et al., 1995; Dodds et al., 1999; Wright et al., 2003; Toledano et al., 2005). Studies on small for gestational age and/or intrauterine growth retardation showed some more consistent results, and a good proportion of them have found statistically significant associations (Kramer et al., 1992; Bove et al., 1995; Gallagher et al., 1998; Wright et al., 2003; Wright et al 2004). Wright et al. (2004) found statistically significant associations with THMs and a measure of mutagenicity, but not with HAAs or MX. Infante-Rivard (2004) found that the association between THMs and intrauterine growth retardation was modified by a metabolic polymorphism, with newborns without the CYP2E1 (G1259C) variant at high risk.

\section{DISCUSSION}

Epidemiological studies on neural tube defects, urinary tract defects and small for gestation age/intra growth retardation have shown the most consistent statistically significant associations with an index of DBPs, but generally the risk estimates are small. The interpretation of the studies is not straight forward because they may not be directly comparable because of differences in DBP mixtures, exposure categories and actually uptake of DBPs due to differences in e.g. ingestion rates, showering, bathing, and swimming. Only few specific DBPs have been studied and THMs have often been used as a marker for other DBPs, since they are often routinely available. However they may not be well correlated with other DBPs and therefore not be a good marker. Sample sizes, and therefore power, have at times been low, particularly when the population was split into exposure categories. Although most studies considered some confounders, (residual) confounding by other water contaminants or other factors related to water intake, cannot always be excluded. Case ascertainment, for outcomes such as spontaneous abortion and certain congenital anomalies is far from straight forward, and for the latter at times anomalies are lumped together with different aetiology, which may be inappropriate. Furthermore, as with many reproductive epidemiological, if the putative agent affects both early pregnancy loss and later birth outcomes such as congenital anomalies, interpretation of later birth outcomes may be more difficult. 
Animal evidence could shed more light on any possible relationships, but doses that have been used were often high, much higher than humans are exposed to and the DBPs are often administered in isolation rather than a mixture (Nieuwenhuijsen et al., 2000b; Gevecker Graves et al., 2001; IPCS 2000). THMs have generally shown no direct evidence of, but e.g. neural tube and craniodefects have been found with administration of dichloroacetic or trichloroacetic acid in rats (Smith et al., 1989), and cardiac malformations have been induced at high doses of dichloroacetic acid (Smith et al., 1992). Andrews et al. (2004) found heart defects and neural tubes with HAAs. Also, Hunter et al. (1996) found changes in neural tube development when they exposed mouse embryos to HAAs. Several chloroacetonitrile compounds have shown an increase in malformations of cardiovascular, digestive, soft tissue and urinogenital systems (Smith et al., 1989; Smith et al., 1988). 2-chlorophenol has also been associated with subfertility and stillbirths (Exon and Koller, 1985).

Epidemiological studies require accurate, precise and biologically relevant exposure estimates, preferably with a large range, which, as can be seen from the review, makes the exposure assessment fairly challenging. There are many different DBPs and uptake of DBPs occurs through various activities and exposure routes (Nieuwenhuijsen et al., 2000a). Routinely collected THM data are often available, models are being developed, but information on personal exposure needs to be collected. Inaccurate and imprecise exposure estimates may lead to loss of power, precision and attenuation in health risk estimates, depending on the type of error model (Armstrong 1998, Nieuwenhuijsen 1997). Researchers generally have to perform some cost-benefit analysis when it comes to choosing an exposure estimate for an epidemiological study. Improving the accuracy of the exposure estimate will generally improve study power and the accuracy and/or precision of the effects of the exposure, but it is likely to come at a financial cost (Armstrong 1996). For example, personal exposure assessment such as biomonitoring of exhaled breath or urine may provide a much more accurate exposure estimate for each study subject than a group estimate such as a water zone mean estimate, at least when a sufficient number of samples are taken. However it may come at a financial cost that is also considerably higher, and may be well out of reach of the study. Relative large sample sizes may be needed for epidemiological studies of birth outcomes, for example for prospective cohort studies, given that many of the outcomes of interest are rare. Case-control studies may require fewer subjects, but the biomonitoring needs to take place after the relevant exposure period and is therefore likely to be less informative than in other study designs with a retrospective exposure assessment. Questionnaire data are also likely to be less accurate than in a prospective design. An important consideration is also whether the study sets out to find an association between a DBP and a certain outcomes, or, in addition, it also attempts to quantify the exposure/dose-response relationship, if any. In addition, one should keep in mind any policy implications, in particular risk management. DBP water zone levels can be regulated while, for example, personal activities cannot be. So what would be an efficient exposure assessment design? Would a study with a small number of subjects and a very accurate exposure estimate e.g. a biomarker in urine be as efficient and informative as a study with a large number of subjects and with a less accurate exposure estimate e.g. water zone estimate?

Some epidemiological studies have used water zone estimates, based on routinely collected THM measurements and/or models, as exposure index (e.g. Kramer et al., 1992, Bove et al., 1995, Gallagher et al., 1998, Dodds et al., 1999). Others have used water zones estimates in combination with personal exposure characteristics (e.g. Savitz et al., 1995, Waller et al., 1998, Klotz and Pyrch 1999). Some questions have been raised on the interpretation of health risk estimates (e.g. odds ratios and relative risks from exposure-response relationships estimating the risk per unit exposure) when using only the mean THM estimates of the water zones as an exposure index. It was suggested 
that information on personal uptake of THMs is needed to interpret, or improve the interpretation of, health risk estimates because of possible exposure misclassification (Swan and Waller, 1998).

In epidemiological studies using personal estimates, the variability in these personal activities may lead to measurement error, and therefore attenuation of health risk estimates, under the so called Classical error model (Armstrong 1998). Under the Classical error model the average of many replicate measurements would equal the true exposure and the degree of attenuation is equal to the coefficient of reliability. Attenuation is less likely to occur when a group estimate such as the mean estimates of water zones are used because the Berkson error model may apply. The Berkson error applies when an approximate exposure (proxy) is used for many subjects in the study; the true exposures vary randomly about this proxy (Armstrong, 1998). In linear, and often in log-linear models, Berkson error is generally unlikely to lead to attenuation in health risk estimates, only to less precise ones, which may reduce study power (Armstrong 1998, Armstrong 1996). The efficiency in this case is equal to the proportion of variance of personal uptake explained by water zone means i.e. the square of the correlation between personal uptake and water zone mean (Armstrong 1996). When designing and interpreting epidemiological studies these issues should be considered.

While the available evidence suggests that the risks, if any, are small, the large numbers of people exposed to chlorinated water supplies means that the population attributable risk is potentially high. The inability to eliminate the possibility that other risk factors or possible biases might explain these small excess risks, coupled with the insufficient animal data to evaluate the biological mechanisms by which these agents may exert teratogenic and other birth effects, makes interpretation of such small elevations in risks difficult. Moreover, many of these apparent associations have been seen at TTHM levels well below the established maximum standards, currently at $100 \mu \mathrm{g} \mathrm{I}^{-1}$ in the UK and US. Reducing chlorination by products concentrations further while still using chlorine becomes increasingly difficult. It must be remembered that the public health benefits of chlorination in terms of microbiological safety far exceed the potential health risks, but alternatives to chlorination should and are being explored e.g. the use of ozone.

Further epidemiological studies are recommended for reproductive outcomes such as (low) birth weight, still birth, spontaneous abortion and birth defects e.g. heart defects, cleft lip, respiratory defects, urinary tract defects, neural tube defect and central nervous system defects, and for studies of adult male fertility based on the current available toxicological and epidemiological evidence. Such studies should use appropriate designs such as a cohort study design for more common outcomes, or case-control study design for the rarer outcomes, with sufficient sample sizes, good case ascertainment, inclusion of relevant confounders, and in-depth exposure assessment (including repeated exposure measures). In practice this may not always be practicable, partly due to the cost involved, but some of these issues could be addressed in subsets of the populations. Also cultural and water treatment differences should be considered. Large registry based studies may also be useful, but a larger effort should be focused on the exposure assessment (see below) and potential for bias and confounding.

Future epidemiological studies will remain relatively crude until exposure assessment improves. Some of the factors that need to be considered are spatial and temporal variability in individual and total THMs and other by products, correlation between different substances, large samples where feasible, the relative contribution of different exposure routes (inhalation, ingestion, dermal absorption), consumption patterns (including tap water and bottled water, hot and cold drinks, and food) and daily activities including showering, bathing and swimming. Although it is unlikely that a single study 
could be carried out taking into account all these factors, future studies need to try and minimise the potential for bias from these sources, possibly by carrying out more detailed exposure characterisation among a subset of the population. This should lead to a better understanding of the distribution and determinants of uptake of chlorination by products, and the design of statistical models to predict dose estimates for epidemiological and risk assessment studies. Furthermore, more effort should be made characterise the mixture of by-products in the study area, which could be very helpful for the interpretation of previous and future epidemiological studies.

\section{ACKNOWLEDGEMENTS}

The review is an update on a previously published review: Nieuwenhuijsen MJ, Toledano MB, Eaton NE, Elliott P and Fawell J. (2000) Chlorination disinfection by-products in water and their association with adverse reproductive outcomes: a review Occup Environ Med 57: 73-85. Further discussion on important issues for epidemiological studies can be found there.

Reproductive effects of chlorine dioxide treatment have not been discussed due to the small number of studies

\section{REFERENCES:}

Andrews JE, Nichols HP, Schmid JE, Mole LM, Hunter ES 3rd, Klinefelter GR.

Developmental toxicity of mixtures: the water disinfection by-products dichloro-, dibromo- and bromochloro acetic acid in rat embryo culture. Reprod Toxicol. 2004 Nov;19(1):111-6.

Armstrong BG. (1996) Optimizing power in allocating resources to exposure assessment in an epidemiologic study. Am J Epidemiol , 144(2):192-7

Armstrong BG. (1998) Effect of measurement error on epidemiological studies of environmental and occupational exposure. Occup Environ Med., 55:651-656

Aschengrau A, Zierler S and Cohen A. (1989) Quality of community drinking water and the occurrence of spontaneous abortion. Arch Environ Health, 44:283-289

Aschengrau A, Zierler S, Cohen A. (1993) Quantity of community drinking water and the occurrence of late adverse pregnancy outcomes. Arch Environ Health, 48:105-113.

Bove FJ, Fulcomer MC, Klotz JB, Esmart J, Dufficy EM, Savrin JE. (1995) Public drinking water contamination and birth outcomes. Am J Epidemiol, 141: 850-862.

Bove F, Shim Y, Zeitz P. (2002) Drinking water contaminants and adverse pregnancy outcomes: a review. Environ Health Perspect., 110 Suppl 1:61-74.

Cedergren MI, Selbing AJ, Lofman O and Kallen B. (2002) Chlorination byproducts and nitrate in drinking water and risk of congenital cardiac defects. Environ Res, Section A, 89:124-130

Dodds L, King W, Woolcott C and Pole J. (1999) Trihalomethanes in public water supplies and adverse birth outcomes. Epidemiology, 3:233-237

Dodds L and King WD. (2001) Relation between trihalomethane compounds and birth defects. Occup Environ Med, 58:443-446

Dodds L, King W, Allen AC, Armson A, Fell DB and Nimrod C. (2004) Trihalomethanes in public water supplies and risk of stillbirth. Epidemiology, 15:179-186

Exon JH, Koller LD. (1985) Toxicity of 2-CP, 2,4,DCP and 2,4,6-TCP. In: Jolley RL et al (eds). Water Chlorination, chemistry and environmental impact and health effects. Vol 5. Chelsea. $\mathrm{MI}$, Lewis Publishers.

Gallagher MD, Nuckols JR, Stallones L and Savitz DA. (1989) Exposure to trihalomethanes and adverse pregnancy outcomes. Epidemiology, 9:484-489

Gevecker Graves C, Matanoski GM and Tarfdiff RG. (2001) Weight of evidence for an association between adverse reproductive and developmental effects and exposure to disinfection byproducts: a critical review. Reg Tox and Pharm, 34:103-124

Hunter ES III, Roger EH, Schmid JE and Richard A. (1996) Comparative effects of haloacetic acids in whole embryo culture. Teratology, 54:57-64

Hwang B-F and Jaakkola JJK. (2003) Water chlorination and birth defects: a systematic review and meta-analysis. Arch Environ health, 58:83-91

Hwang B-F, Magnus P and Jaakkola JJK. (2002) Risk of specific birth defects in relation to chlorination and the amount of natural organic matter in the water supply. Am J Epidemiol, 156:374-382

Infante-Rivard C. (2004) Drinking water contaminants, gene polymorphisms and fetal growth. 
Environ Health Perspec, 112:1213-1216

IPCS. (2000) Disinfectants and disinfectant by-products. Environmental Health Criteria 216, WHO, Geneva.

Jaakkola JJK, Magnus P, Skrondal A, Hwang B-F, Becher G and Dybing E. (2001) Foetal growth and duration of gestation relative to water chlorination. Occup Environ Med, 58:437-442

Kallen BAJ and Robert E. (2000) Drinking water chlorination and delivery outcome-a registrybased study in Sweden. Reproductive Toxicology, 14:303-309

Kanitz S, Franco Y, Patrone V, Caltabellotta M, Raffo E, Riggi C, Timitilli D, Ravera G. (1996) Association between drinking water disinfection and somatic parameters at birth. Environ Health Persperc, 104:516-520.

King WD, Dodds L and Allen AC. (2000) Relation between stillbirth and specific chlorination byproducts in public water supplies. Environmental Health Perspectives, 108: 883-886

King WD, Dodds L, Allen AC, Armson BA, Fell D and Nimrod C. (2005) Haloaceticacids in drinking water and risk for stillbirth. Occup Environ Med,62:124-127.

Klotz JB and Pyrch LA. (1999) Neural tube defects and drinking water disinfection by-products. Epidemiology,10:383-390

Kramer MD, Lynch CF, Isacson P, Hanson JW. (1992) The association of waterborne chloroform with intrauterine growth retardation. Epidemiology, 3:407-413

Magnus P, Jaakkola JJK, Skrondal A, Alexander J, Becher G, Krogh T and Dybing E. (1999) Water chlorination and birth defects. Epidemiology, 10: 513-517

Nieuwenhuijsen MJ, Northstone K and Golding J. (2002) Swimming and birth weight. Epidemioly, 13:725-728

Nieuwenhuijsen MJ, Toledano MB and Elliott P. (2000) Uptake of chlorination disinfection byproducts; a review and a discussion of its implications for epidemiological studies. $J$ Expos Anal Environ Epidemiol , 10: 586-599

Nieuwenhuijsen MJ, Toledano MB, Eaton NE, Elliott P and Fawell J. (2000) Chlorination disinfection by-products in water and their association with adverse reproductive outcomes: a review Occup Environ Med 57: 73-85.

Reif JS, Hatch MC, Bracken M, Holmes LB, Schwetz BA and Singer PC. (1996) Reproductive and development effects of disinfection by-products in drinking water. Environ Health Perspec, 104: 1056-1061

Richardson S. (1998) Drinking water disinfection by-products. In: Encyclopedia of environmental analysis and remediation, John Wiley \& Sons, Inc, ISBN 0-471-11708-0.

Rook JJ. (1974) Formation of haloforms during chlorination of natural waters. J Soc for Water Treatment and Examination, 23:234-243.

Savitz DA, Andrews KW, Pastore LM. (1995) Drinking water and pregnancy outcome in Central

North Carolina: Source, amount and trihalomethane levels. Environ Health Persperc, 103:592-596.

Shaw GM, Ranatunga D, Quach T, Neri E, Correa A and Neutra R. (2003) Trihalomethane exposures from municipal water supplies and selected congenital malformations. Epidemiology, 14:191-199.

Smith MK, Randall JL, Read JL, Stober JA. (1992) Developmental toxicity of dichloroacetate in the rat. Teratology, 46: 217-223.

Smith MK, Randall JL, Stober JA. (1989) Teratogenic activity of trichloroacetic acid in the rat. Teratology, 40: 445-451.

Smith MK, Randall JL, Stober JA, Read EJ. (1989) Developmental toxicity of dichloroacetonitrile: a by-product of drinking water disinfection. Fundamen Appl Toxicol, 12: 765-772.

Smith MK, Randall JL, Tocco DR, York RG, Stober JA, Read EJ. (1989) Teratogenic effects of trichloroacetonitrile in the Long-Evans rat. Teratology, 38: 113-120.

Toledano MB, Nieuwenhuijsen MJ, Bennet J, Best N, Whithaker H, Cockings S, Fawell J, Jarup L, Briggs D and Elliott P. (2005) Chlorination disinfection by-products and adverse birth outcomes in Great Britain: birthweight and still birth. Environ Health Perspec, 113: 225-232

Villanueva CM, Cantor KP, Cordier S, Jaakkola JJ, King WD, Lynch CF, Porru S, Kogevinas M. (2004) Disinfection byproducts and bladder cancer: a pooled analysis. Epidemiology, 15(3):357-67

Waller K, Swan SH, DeLorenze G, Hopkins B. (1998) Trihalomethanes in drinking water and spontaneous abortion. Epidemiology, 9: 134-140.

Waller K, Swan S, Windham GC and Fenster L. (2001) Influence of exposure assessment 
methods on risk estimates in an epidemiologic study of trihalomethane exposure and spontaneous abortion. J Expos Anal Environ Epidemiol, 11: 522-531

Wright JM, Schwartz J and Dockery DW. (2003) Effect of trihalomethane exposure on fetal development. Occup Environ Med, 60:173-180

Windham GC, Waller K, Anderson M, Fenster L, Mendola P and Swan S. (2003) Chlorination byproducts in drinking water and menstrual cycle function. Environ Health Perpec, 111:935-941

Wright JM, Schwartz J and Dockery DW. (2004) The effect of disinfection by-products and mutagenic activity on birth weight and gestational duration. Environ Health Perspect. 112(8):920-5.

Yang C-Y, Cheng B-H, Tsai S-S, Wu T-N, Lin M-C and Lin K-C. (2000a) Association between chlorination of drinking water and adverse pregnancy outcome in Taiwan. Environmental Health Perspectives, 108: 765-768

Yang C-Y, Cheng B-H, Tsai S-S, Wu T-N, Hsu T-Y and Lin K-C. (2000b) Chlorination of drinking water and sex ratio at birth in Taiwan. J Tox Environ Health, 60:471-476

Yang C-Y. (2004) Drinking water chlorination and adverse birth outcomes in Taiwan, Toxicology, 198(1-3): 249-254. 